\title{
LA INFLUENCIA DE LA INDUSTRIA ALIMENTARIA EN LA ÉTICA DEL NUTRIÓLOGO. ¿A QUIÉN LE CONVIENE QUÉ?
}

THE INFLUENCE OF THE FOOD INDUSTRY ON THE ETHICS OF THE NUTRIOLOGIST. TO WHOM IS IT SUITABLE?

\author{
Ceballos-Suárez Silvia Cristina*, Castillo-Hernández Karen G.*
}

* Escuela de Ciencias de la Salud de la Universidad Marista de Mérida, México.

Citation: Ceballos-Suárez S.C., Castillo Hernández K.G. (2019) La influencia de la industria alimentaria en la ética del nutriólogo. ¿A quién le conviene qué? Revista Salud Pública y Nutrición, 18 (4), 17-22.

Editor: Esteban G. Ramos Peña, Dr. CS., Universidad Autónoma de Nuevo León, Facultad de Salud Pública y Nutrición, Monterrey Nuevo León, México. Copyright: (02019 Ceballos-Suárez S.C. et al. This is an open-access article distributed under the terms of Creative Commons Attribution License [CC BY 4.0], which permits unrestricted use, distribution, and reproduction in any medium, provided the original author and source are credited.

Competing interests: The authors have declared that no competing interests exist.

DOI: https://doi.org/10.29105/respyn18.4-3

Recibido: 20 de junio 2019;

Aceptado: 08 de octubre 2019

Email: nutricalli@gmail.com 


\title{
LA INFLUENCIA DE LA INDUSTRIA ALIMENTARIA EN LA ÉTICA DEL NUTRIÓLOGO. ¿A QUIÉN LE CONVIENE QUÉ?
}

\author{
Ceballos Suárez Silvia Cristina*, Castillo Hernández Karen G.* \\ * Escuela de Ciencias de la Salud de la Universidad Marista de Mérida, Yucatán, México.
}

\section{RESUMEN}

Introducción. La revolución industrial fue pilar del modelo económico capitalista, surgido a principios del siglo XIX. En este modelo se tiende a maximizar las ganancias, fomentando un consumo cada vez mayor de productos. La industria alimentaria ha seguido esta misma tendencia afectando la salud de la población a través del sobreconsumo de productos de baja calidad nutrimental. El profesionista de la nutrición también ha sido involucrado en las estrategias del mercado generando incertidumbre sobre su ética profesional. Objetivo: Reflexionar sobre la responsabilidad ética que tiene el nutriólogo frente a las estrategias de sobreconsumo que promueve la industria alimentaria. Conclusiones: El profesionista de la nutrición podría tener un papel más activo en la regulación de las normativas de promoción de la industria alimentaria siendo su deber anteponer el bienestar de las personas frente a los intereses económicos.

Palabras Clave: Capitalismo, industria alimentaria, ética profesional.

\section{ABSTRACT}

Introduction: The industrial revolution was a pillar of the capitalist economic model, which emerged in the early nineteenth century. This model tends to maximize profits, promoting an increasing consumption of products. The food industry has followed this same trend affecting the health of the population through the overconsumption of products of low nutritional quality. Dietitians have also been involved in market strategies calling into question professional ethics. Objective: To reflect on the ethical responsibility that nutritionists have against the overconsumption strategies promoted by food industry. Conclusions: Dietitians could have a more active role in the regulation of promotions of the food industry, being his duty to put the welfare of the people before any other interest.

Key words: Capitalism, food industry, health professional ethics. 


\section{Introducción}

El capitalismo es un modelo económico que surge con la revolución industrial a principios del siglo XIX. En el capitalismo, el trabajo artesanal fue reemplazado por un trabajo especializado, mecanizado y colectivo (asalariado) controlado por "la empresa". Además, la mecanización del trabajo permitió vender más a un precio más bajo, eliminando a las empresas más pequeñas que no son capaces de integrar la tecnología y ser competitivas (Gouvener et al., 2005).

Las empresas en el afán de incrementar sus ganancias, eliminan o absorben a otras empresas más débiles, concentrando cada vez más el capital, monopolizando la producción, a los asalariados y encapsulando poco a poco el poder en sus manos. La concentración del capital favorece acuerdos entre iguales fijando precios monopólicos y manteniendo la competencia mediante sus ejes de publicidad e innovación. Debido a lo anterior y en la búsqueda por multiplicar sus ganancias, éstas invierten en otras ramas de producción, diversificándose y perpetuando la misma dinámica de negocio (Gouvener et al., 2005). Cuando algunas de estas grandes empresas conocidas como multinacionales cruzan las fronteras de los países ejerciendo el mismo ciclo, el poder que tienen es tal, que son capaces de interferir en las agendas políticas.

México ha sido sede de grandes multinacionales de la industria alimentaria, lo anterior es relevante porque nuestro país ocupa uno de los primeros lugares a nivel mundial en consumo de algunos productos procesados como las bebidas azucaradas. Esto no es independiente de los problemas de obesidad que tiene la nación, que a pesar de ser conocidos están lejos de ir disminuyendo. Por ejemplo, las prevalencias de sobrepeso y obesidad han aumentado de acuerdo con los resultados de la ENSANUT MC 2016 que compara los datos con respecto a la ENSANUT 2012. Es conocido que la industria alimentaria, financia investigación, patrocina eventos relacionados con la salud y a los profesionistas los involucra en sus estrategias de mercado (Barquera et al., 2018). En el presente trabajo las autoras, Nutriólogas, apoyan la tesis de que el modelo económico actual es un determinante de las decisiones respecto a la salud poblacional lo que a su vez ha transgredido la ética del profesional de la salud.
El capitalismo y el crecimiento de la industria alimentaria

Según el reporte PROMEXICO (2018), la industria de alimentos procesados representa el 3.9\% del PIB liderado por las categorías de panadería y confitería. Los productos listos para consumir y ultraprocesados han incrementado sus ventas y se espera que la producción crezca durante el periodo 2018-2021 (PROMEXICO, 2018). Con este crecimiento, aumenta también la disponibilidad de azúcar simple, grasa saturada, sodio y energía, factores que contribuyen al exceso de peso poblacional y a sus consecuencias negativas a la salud como lo son la diabetes, las enfermedades cardiovasculares, el cáncer, por mencionar algunas. (Monteiro et al., 2018).

En el modelo económico capitalista, las leyes de mercado determinan el crecimiento de las empresas, nacionales o multinacionales, que tienen la capacidad productiva para generar más a un menor costo. Estas leyes también determinan cuáles negocios desaparecen por ser ineficientes, sustituyendo a los productores primarios locales. Entonces, la producción local de alimentos disminuye, incrementándose las importaciones. (NavarreteReynoso et al., 2015).

Lo anterior se explica debido a diversos factores que reorganizan el mercado como la reestructuración familiar, en la que ambos padres trabajan, disminuyendo el tiempo para la preparación de alimentos e incrementando la demanda de alimentos procesados. Además, en las crisis económicas y en la pobreza, situación en la que viven más de 50 millones de personas en el país, se destina la mayor parte del ingreso a las necesidades básicas como la alimentación (FAO, 2018). Otros factores que han contribuido a la dinámica actual del mercado alimentario son: el crecimiento urbano que disminuye la disponibilidad de la tierra cultivable, los canales de distribución de alimentos ampliados a tiendas de autoservicio que han absorbido o eliminado a las pequeñas empresas locales y la ya mencionada monopolización de los precios establecidos por las grandes empresas de alimentos. (Navarrete- Reynoso et al., 2015).

Salud vs industria alimentaria. A partir del escenario planteado, la Estrategia mundial sobre régimen alimentario, actividad física 
y salud propuesta por la OMS (2004), alienta a la industria alimentaria a realizar cambios. Concretamente, la reducción de las grasas saturadas, los ácidos grasos trans, los azúcares libres y la sal en los productos alimentarios procesados; así como la reducción del tamaño de las porciones y el aumento de opciones alimentarias innovadoras, saludables y nutritivas. También recomienda la revisión de la publicidad, promoviendo información nutricia veraz y de fácil comprensión. Sin embargo, existe oposición por parte de la industria, esquivando las demandas a través de una autoregulación ineficiente o responsabilizando al consumidor de las elecciones alimentarias (Ludwin et al., 2008).

En nuestro país, se han propuesto políticas públicas para modificar el ambiente comercial y facilitar comportamientos saludables, así como empoderar al consumidor para elegir de una manera informada los alimentos que consume (SSA, 2010). La sociedad civil ha tratado de contribuir a informar a la población acerca del contenido nutricional de productos alimentarios, además de advertir sobre las campañas con publicidad engañosa (El poder del consumidor A.C., 2019). Sin embargo, es necesario preguntarse si las acciones encaminadas a disminuir el exceso de peso están siendo efectivas, debido a que no se ha evidenciado una mejoría en el estado de nutrición de los mexicanos de acuerdo a la ENSANUT MC 2016, al contrario, la diabetes y el sobrepeso en adultos siguen aumentando (Shamah et al., 2016). Por lo tanto, resultan cuestionables las políticas públicas, los esfuerzos de la sociedad civil y la autoregulación de la industria alimentaria. ¿Acaso la disponibilidad de alimentos poco saludables no es cada vez mayor? ¿El consumo de alimentos ultraprocesados no ha aumentado?

La evaluación de los hábitos de los mexicanos refiere que el consumo de alimentos ultraprocesados, es común. Al menos 5 de cada 10 escolares y adolescentes consumen frecuentemente botanas y frituras. El 34\% de los preescolares consumen comida rápida. Es alarmante mencionar que 9 de cada 10 mexicanos consumen bebidas azucaradas, destacando el consumo de refresco de cola en 7 de cada 10. Además, al menos el $50 \%$ de la población pediátrica consume bebidas azucaradas de forma cotidiana (Arvizú et al., 2015). Aunado a esto, el INEGI (2016) reportó que el 7.7\% de los ingresos de los mexicanos se destinan al consumo de alimentos y bebidas fuera del hogar y de este gasto, el $2.4 \%$ se destina al consumo de bebidas.

En el año 2014 se estableció en nuestro país un impuesto a las bebidas azucaradas, como una medida recomendada por la OMS para disminuir su consumo. Los resultados de esta intervención publicados dos años después, mencionan que la compra de estos productos disminuyó $8 \%$ durante ese periodo, también se reportó un incremento del $2.1 \%$ en las ventas de las bebidas no gravadas (Colchero et al., 2017). Con respecto a los alimentos densamente energéticos (275 kcal/100gr) que no son considerados de consumo básico, también se estableció una medida recaudatoria del 8\%. En la evaluación posterior a un año, se observa una disminución en la compra del $10.2 \%$ en el nivel socioeconómico bajo, del $5.8 \%$ en el nivel medio y sin cambios en el estrato más alto (Batis et al., 2016).

Dos de las grandes multinacionales de bebidas azucaradas, respondieron al impuesto establecido con estrategias de mercadotecnia multimillonarias que vinculan el consumo de sus productos con emociones positivas, dirigidas en gran medida a la población joven y a aquella que vive en las comunidades más alejadas y marginadas, las cuales difícilmente cuentan con atención a la salud ni acceso a información sobre los daños que ocasiona el consumo de bebidas embotelladas. Otra medida, fue financiar investigaciones que diluyen la evidencia que vincula la obesidad con el consumo de estos productos, sin embargo, se identificó a través de una revisión sistemática que los resultados financiados por la industria encontraron una asociación 5 veces menor que aquellos estudios que no fueron financiados (Du et al., 2018) y (Bes-Rastrollo et al., 2013).

La industria alimentaria también ha tenido un interés en el desarrollo de alimentos modificados en grasas, azúcar o energía, así como de los alimentos funcionales y nutracéuticos debido a la creciente demanda de estos productos. Así, el profesional de la nutrición se ha involucrado en la industria mediante la investigación, promoción y publicidad de alimentos, etiquetado nutricional y educación al consumidor (Rodríguez y Ureña, 2007). En este sentido, la industria alimentaria financia investigaciones para el desarrollo de nuevos productos, que "ayuden a mejorar la salud de la 
población", sin embargo, debido a casos como el mencionado sobre las bebidas azucaradas, el apoyo es cuestionable.

\section{Conflictos éticos}

Un conflicto de interés se refiere a las situaciones en las cuales consideraciones tanto financieras como de otra índole, podrían comprometer un interés por la salud del paciente o de la población por un interés personal o institucional. En investigación, un conflicto de interés puede comprometer el juicio del investigador y conducirlo a reportar resultados falsos o modificados (Herrera, 2016). Recientemente se publicó que la empresa Coca-Cola ha financiado 8 millones de euros a organizaciones científicas y médicas, los cuales se han destinado a la investigación y a la organización de congresos (Ansede, 2018), lo anterior es importante porque es contradictorio encontrar stands de la marca en los congresos de nutrición ya que como se ha evidenciado, el consumo excesivo de refrescos y bebidas azucaradas es causante de obesidad.

Lo peligroso de recibir financiamiento de las grandes empresas es responder a la pregunta planteada iA quién le conviene qué? Debido a que el desarrollo de productos saludables debería ser un interés común como lo recomiendan las directrices mundiales, sin embargo ¿Por qué las etiquetas son engañosas? ¿Por qué se le pone la etiqueta de "integral" a los productos, pero no la advertencia de que tienen jarabe de alta fructuosa o grasas trans? ¿Por qué si las grandes empresas tienen asesoría de expertos, un producto etiquetado como saludable no lo es en realidad? ¿Será que es caro producir alimentos saludables o será que nos quieren enfermos?

\section{La ética del nutriólogo}

La Ley reglamentaria del artículo $5^{\circ}$ constitucional relativo al ejercicio de las profesiones establece sanciones para todo aquel que infrinja las disposiciones establecidas por la misma (Cámara de Diputados del H. Congreso de la Unión, 2018). Sin embargo, a diferencia de las normas legales, en el caso de las normas éticas no existe una sanción establecida por alguna autoridad ante su incumplimiento. La ética depende exclusivamente del conocimiento del bien, de la conciencia, voluntad y libertad del profesionista para adherirse a un código ético de conducta.
La Asamblea General de las Naciones Unidas (1948) en la declaración de los derechos humanos menciona que "Toda persona tiene derecho a un nivel de vida adecuado que le asegure, así como a su familia, la salud y el bienestar, y en especial la alimentación...". El código de ética del nutriólogo propuesto por el Colegio Mexicano de Nutriólogos exhorta al profesionista en los artículos $3^{\circ}, 8^{\circ}$ y $22^{\circ}$ a respetar los derechos humanos de sus pacientes y sociedad en general, así como a conducirse con la verdad sin anteponer sus intereses (Colegio Mexicano de Nutriólogos A.C. 1999).

Ante el poder y la influencia que tiene la industria alimentaria, es imperativo preguntarse ¿Cuál es la responsabilidad del nutriólogo para con la sociedad ante un escenario como el que se ha descrito? La versatilidad de la profesión permite realizar acciones desde diferentes escenarios. Entre las acciones que se proponen están: evitar el conflicto de interés prescindiendo de patrocinios incongruentes en eventos formativos, investigaciones, en la consulta privada, en proyectos comunitarios, privilegiando a productores locales $u$ organizaciones que fomenten la alimentación saludable (Barquera et. al, 2018). El nutriólogo debe ser capaz de empoderar a las comunidades para que puedan realizar elecciones alimentarias que contribuyan a su salud con información fehaciente, además de proporcionar opciones de alimentos locales que contribuyan a la salud y a la economía mexicana. El nutriólogo también debe ser congruente, una de las tareas más difíciles de realizar. Ante el privilegio de contar con un grado de estudios en el área de la salud, la postura del profesional de la nutrición no puede ser expectativa, es necesario "hacer". Si bien el modelo económico está en contra, es muy importante recordar que el actuar siempre favoreciendo la salud de nuestros pacientes o de las comunidades en las que influimos es nuestra responsabilidad ética.

\section{Conclusiones}

La conveniencia de la industria alimentaria por incrementar su capital parece dejar en desventaja al profesional de la salud, su capacidad financiera le permite realizar una inteligente y efectiva publicidad, lo cual le ha permitido colocar sus productos ultraprocesados de forma cotidiana y excesiva en las mesas de los mexicanos, siendo los productos alimenticios de baja calidad más accesibles para aquellos que viven en situaciones de pobreza y 
vulnerabilidad. Además, mediante la innovación de productos, confunden al consumidor promoviendo como alimentos "saludables" aquellos que no lo son. Lo anterior no es regulado de forma efectiva debido a que la industria alimentaria también ha sido capaz de corromper políticas públicas, instituciones de salud y al mismo profesional de la salud.

¿A quién le conviene qué? Es una pregunta que debe ser abordada desde la formación profesional, no solamente dando a conocer los principios éticos, también incentivando la creatividad del futuro profesionista para proponer acciones en pro del bienestar de la población. En la era de las redes sociales, éstas pueden ser un puente de comunicación para difundir información veraz, orientar a la población en la lectura de etiquetas y denunciar a los productos con publicidad engañosa. El nutriólogo debe formarse para ejercer su profesión bajo principios éticos, sumar acciones para que la población pueda realizar elecciones alimentarias que beneficien su salud, proponer políticas públicas, promover opciones saludables de productores locales, contribuir a la formación ética de los nuevos profesionistas y predicar con el ejemplo. La conducta ética de los profesionales, puede sumar y contribuir al bienestar de la sociedad.

\section{Bibliografía}

Andese M. (12 de septiembre de 2018). Coca-cola ha pagado ocho millones de euros a asociaciones médicas y científicas en España. El país. Recuperado de https://elpais.com/elpais/2018/09/07/ciencia/1536332 059_096535.html

Arvizú O., Polo E. y Shamah T. (2015). Qué y cómo comemos los mexicanos. Consumo de alimentos en la población urbana. México: Grafia Editores.

Asamblea General de las Naciones Unidas. (1948). Declaración universal de los derechos humanos.

Barquera S, García-Chávez C, Navarro Rosenblat D, Uauy R, Pérez-Escamilla R, Martorell R. (2018). Postura de la Sociedad Latinoamericana de Nutrición (SLAN) sobre el manejo de conflicto de intereses. Salud pública de México, 60 (5), 592-597.

Batis C, Rivera JA, Popkin BM, Taillie LS. (2016). FirstYear Evaluation of Mexico's Tax on Nonessential Energy-Dense Foods: An Observational Study. PLoS Med, 13(7):e1002057.
Bes-Rastrollo M, Schulze MB, Ruiz-Canela M, MartinezGonzalez MA. (2013). Financial Conflicts of Interest and Reporting Bias Regarding the Association between Sugar-Sweetened Beverages and Weight Gain: A Systematic Review of Systematic Reviews. Stuckler D, editor. PLoS Med, 10(12):e1001578. [PMC free article] [PubMed]

Cámara de Diputados del H. Congreso de la Unión (2018). Ley Reglamentaria del artículo $5^{\circ}$ Constitucional, relativo al ejercicio de las profesiones en la ciudad de México.

Colchero, M. A., Rivera-Dommarco, J., Popkin, B. M., \& Ng, S. W. (2017). In Mexico, Evidence Of Sustained Consumer Response Two Years After Implementing A Sugar-Sweetened Beverage Tax. Health affairs (Project Hope), 36(3), 564-571.

Colegio Mexicano de Nutriólogos A.C. (1999). Código de ética profesional del nutriólogo.

Du, M., Tugendhaft, A., Erzse, A., \& Hofman, K. J. (2018). Sugar-Sweetened Beverage Taxes: Industry Response and Tactics. The Yale journal of biology and medicine, 91(2), 185-190.

FAO, FIDA, UNICEF, PMA y OMS. (2018). El estado de la seguridad alimentaria y la nutrición en el mundo. Fomentando la resiliencia climática en aras de la seguridad alimentaria y la nutrición. FAO, Roma.

Gutiérrez J., Rivera J., Shamah T., Oropeza C. y Ávila M. (2012) Encuesta Nacional de Salud y Nutrición, 2012. Resultados Nacionales. Recuperado de https://ensanut.insp.mx/informes/ENSANUT2012Res ultadosNacionales.pdf

Gouvener J. (2005). Los fundamentos de la economía capitalista. Una introducción al análisis económico marxista del capitalismo contemporáneo. Madrid, España:Maia Editores.

Herrera M. (2016). Conflicto de interés o intereses en conflicto, transparencia en la obtención y análisis de datos científicos. An Venez Nutr, 29(1), 31-36.

Instituto Nacional de estadística y geografía. (2016). Encuesta nacional de ingresos y gastos de los hogares (ENIGH) 2016. Recuperado de http://www.beta.inegi.org.mx/proyectos/enchogares/r egulares/enigh/nc/2016/default.html

Ludwing D.,Nestle M.(2008). Can the food industry play a constructive role in the epidemic of obesity? JAMA, 300(15), 1808-1811. 
Monteiro, C. A., Cannon, G., Moubarac, J., Levy, R. B., Louzada, M. L. C., \& Jaime, P. C. (2018). The un decade of nutrition, the NOVA food classification and the trouble with ultra-processing. Public Health Nutrition, 2l(1), 5-17. doi:10.1017/S1368980017000234

Navarrete- Reynoso R., Arredondo-Hidalgo M., GónzalezRosas E. (2015). Revisión de la evolución de la industria alimentaria en México. Revista de investigación y desarrollo, 1 (1), 7-17.

OMS. (2004). Estrategia mundial sobre régimen alimentario, actividad física y salud fomenta la formulación y promoción de políticas, estrategias y planes de acción nacionales para mejorar la dieta y alentar la actividad física. Recuperado de http://www.who.int/dietphysicalactivity/childhood_pr ivate_sector/es/

Cabada, Xaviera (2019/dic/9). Radiografía de chorizo de pavo fud. El poder del consumidor. Recuperado de: https://elpoderdelconsumidor.org/2019/12/radiografia -de-chorizo-de-pavo-fud/

PROMEXICO (2018). La industria de alimentos procesados. Febrero 2018. Recuperado de http://www.promexico.gob.mx/documentos/sectores/p resentacion-alimentos-procesados.pdf

Rodríguez S. y Ureña M. (2007). El rol del nutricionista en la industria alimentaria: el caso Costa Rica. Revista de Salud Pública y Nutrición, 8 (1), 1-4. Recuperado de http://respyn.uanl.mx/index.php/respyn/article/view/1 $84 / 166$

Secretaria de salud. (2010). Acuerdo nacional para la salud alimentaria. Estrategia contra el sobrepeso y la obesidad. Recuperado de http://activate.gob.mx/Documentos/ACUERDO\%20N ACIONAL\%20POR\%20LA\%20SALUD\%20ALIME NTARIA.pdf

Shamah T., Cuevas L., Dommarco J. y Hernández M. ( 2016). Encuesta Nacional de Salud y Nutrición de Medio Camino, 2016. Resultados Nacionales. Recuperado de http://www.promocion.salud.gob.mx/dgps/descargas1 /doctos_2016/ensanut_mc_2016310oct.pdf 